\title{
Hardware and software complex for researching medical nanoelectrodes
}

\author{
V.L. Kim, S.A. Andreev \\ Institute of Cybernetics \\ TPU \\ Tomsk, Russian Federation \\ kimval11@rambler.ru
}

\begin{abstract}
In medical devices required in electrocardiography, electroencephalography, electromyography, electrooculography, etc. the most important element is the medical electrode intended for measuring biopotentials. The optimal selection of electrodes is possible only after testing them to confirm the claimed manufacturers' parameters or determine the real metrological characteristics. Technical characteristics of measuring instruments produced by domestic producers or supplied by foreign companies are regulated by the State Standard GOST 25995-83. Thus, in accordance with this standard, calibration equipment has to have inherent noise level (basic parameter) up to $15 \mu \mathrm{V}$ in a frequency band up to $10 \mathrm{kHz}$. However, this requirement does not satisfy modern trends of medical instrument engineering in connection with the appearance of new medical nanoelectrodes on the market, including domestic production. A hardware and software complex for researching medical electrodes (HSCRME) was developed at Tomsk Polytechnic University. It allows measuring the parameters of electrode cells: an electrode potential difference; a drift of the electrode potential difference; a noise voltage; an electromechanical noise; an impedance of electrodes; a polarization voltage. The HSCRME includes a measurement analog channel (AMC) and a digital subsystem being a personal computer. The AMC is configured automatically when the user selects the appropriate mode depending on the measured parameter of the electrode. Cross-platform application framework $Q t$ is the software foundation of the HSCRME. Potential possibilities of the HSCRME are determined by the noise of AMC. The noise applied to the input of AMC did not exceed $1 \mu \mathrm{V}(\mathrm{rms})$ in the frequency range $0 \ldots 10 \mathrm{kHz}$. Verification of the complex was carried out by the inductive voltage divider, which is part of State Standard GAT193-2011. This State Standard is stored in VNIIFTRI (Moscow).
\end{abstract}

Keywords - hardware and software complex, medical electrode, metrological characteristics, a noise voltage.

\section{INTRODUCTION}

In medical devices and systems required in electrocardiography, electroencephalography, electromyography, electrooculography, etc. the most important element is the sensor which is the medical electrode intended for measuring biopotentials. The optimal selection of sensors is possible only after testing them to confirm the claimed manufacturers' parameters or determine the real metrological characteristics. Metrological characteristics of electrodes greatly determine potential capabilities and reliability of the results of the electrophysiological methods used for studying the functional state of the human body as a whole and its individual organs as well. Therefore foreign and domestic producers create a large number of electrodes with different physical principles of work, design solutions and specifications. Taking into consideration that this knowledge plays a crucial role in the proper system design of medical devices and systems, an urgent problem is to create calibration equipment or test stands which allow us to determine parameters and characteristics of medical electrodes with high metrological reliability and validity [1-3]. The State Standard GOST 25995-83 regulates technical characteristics of measuring instruments produced by domestic producers or supplied by foreign companies. Thus, in accordance with this standard, calibration equipment has to have inherent noise level (basic parameter) up to $15 \mathrm{mV}$ in a frequency band up to 10 $\mathrm{kHz}$. However, this requirement does not satisfy modern trends of medical instrument engineering in connection with the appearance of new medical nanoelectrodes on the market, including domestic production $[4,5]$.

\section{GENERAL DESCRIPTION.}

A hardware and software complex for researching medical electrodes (HSCRME) was developed at Tomsk Polytechnic University. It allows measuring the parameters of electrode cells according to the State Standard GOST 25995-83:

- an electrode potential difference;

- a drift of the electrode potential difference;

- a noise voltage

- an electromechanical noise;

- an impedance of electrodes;

- a polarization voltage.

The HSCRME (Fig. 1) includes a measurement analog channel (AMC) and a digital subsystem being a personal computer (PC). The measuring amplifier A1 with gain $K_{\mathrm{g} 1}=$ 100 , the programmable amplifier $\mathrm{A} 2$ with gain $K_{\mathrm{g} 2}=1$ and 10 , the programmable amplifier A3 with gain $K_{\mathrm{g} 3}=50$ and 500 , the active low-pass filters LF1 and LF2 with cut-off frequency 75 $\mathrm{Hz}$ and $10 \mathrm{kHz}$, the data acquisition board (DAB) are the basis of the AMC [6]. Repeater (R) is used to reduce the impact of the DC autocorrection circuit on the measured signal at the A1 output. The connection between the lower and upper levels of the system is carried out via an USB. The additional blocks of an analog converter DAC2 and a current generator $\mathrm{CG}$ provide adjusting a constant component $\mathrm{AMC}$ and the given current of electrode cells EC mode (through the analog switch AS) [7]. Voltage from the outputs of the amplifiers A1, A2 and filters LF1, LF2 feeds multiplex inputs $1-4$ of an analog-to-digital converter (ADC) DAB. Control inputs of A2, A3, DAC 2, AS, 


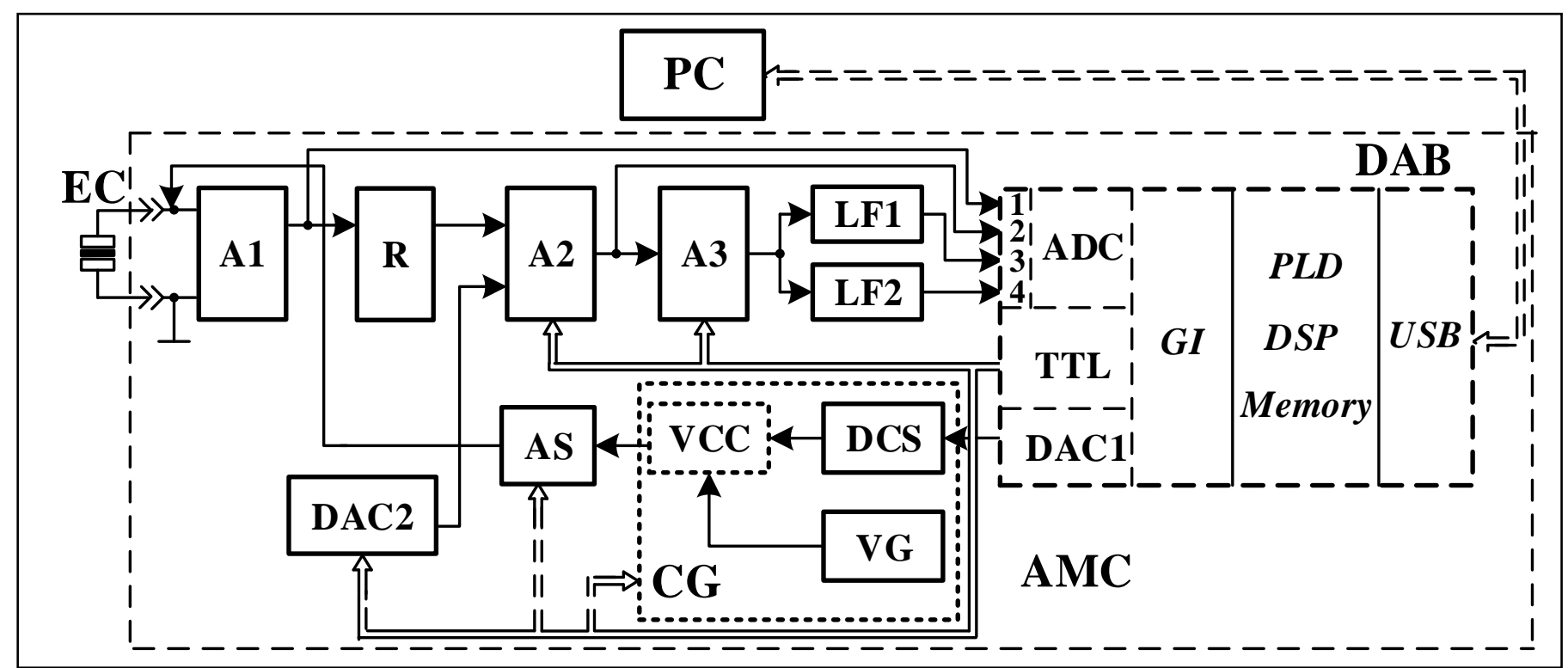

Fig. 1. Schematic diagram of analog measurement channel. EC - electrode cells, A1 - measuring amplifier, R - Repeater, A2 - programmable amplifier, A3 programmable amplifier, LF1 - active low-pass filter, LF2 - active low-pass filter, CG - current generator, DCS - direct current source, VG - voltage generator, VCC - voltage-current converter, AMC - adjusting a constant component, AS - analog switch, DAC2 - digital-to-analog converter, DAB - data acquisition board, ADC - analog-to-digital converter, TTL - transistor-transistor logic (digital outputs), DAC1 - digital-to-analog converter, GI - galvanic isolation unit,

PLD - programmable logic device, DSP - digital signal processor, Memory - dynamic, non-volatile configuration, non-volatile program memory, USB universal serial bus, $\mathrm{PC}$ - personal computer

and CG are connected to the digital TTL outputs of DAB [8]. Digital Signal Processor (DSP) controls, collect and process data. Programmable logic device (PLD) is used in scheme due to the need a large number of discrete input-output ports. Note that $\mathrm{CG}$, made on the basis of the voltage generator (VG) and voltage-current converter (VCC) has two control inputs. The DC voltage from the digital-to-analog converter DAC1 feeds the analog control input of the VG. The frequency of $\mathrm{CG}$ in subbands switched by the digital code is changed by this voltage. Direct current source (DCS) with VCC generates direct current for EC when measured electrodes impedance. Galvanic isolation of ADC, DAC1 digital signal interfaces, and the TLL discrete signals is realized by galvanic isolation unit (GI). Dynamic memory is necessary for the temporary storage of intermediate results, since the speed of the interface may be less than the speed of data acquisition. Non-volatile configuration memory is needed to store rarely changing information. Machine code is stored in non-volatile program memory. All memory types represented as Memory unit on fig. 1 .

The AMC is configured automatically when the user selects the appropriate mode depending on the measured parameter of the electrode, for example:

\section{A. Measurement of an electrode potential difference}

The measurement channel includes amplifiers A1 and A2 with the overall gain $\operatorname{Kg} \Sigma=\operatorname{Kg} 1 \operatorname{Kg} 2=100 \cdot 1=100$. The electrode cell is connected to the input A1. Bias voltage supplied to the auxiliary input A2 from output DAC2 equals zero. In this mode, the program scans only the second ADC channel and outputs the measurement result of an electrode potential difference on a display. Measurement range $-0 \ldots$ $100 \mathrm{mV}$, resolution $-10 \mathrm{mV}$.

\section{B. Measurement of the electrode noise voltage}

The amplifier A3 with gain $\mathrm{Kg} 3=500$ and filter FL2 are added to the measurement channel, the gain of the second amplifier is set to $\mathrm{Kg} 2=10$. Then overall gain equal $\mathrm{Kg} \Sigma=$ $\mathrm{Kg} 1 \mathrm{Kg} 2 \mathrm{Kg} 3=100 \cdot 10 \cdot 500=500000$. Periodic auto correction by the perturbation or it can be called by input voltage (electrode potential) with help of DAC2, is used to reduce the voltage of the constant component in DAC2. The signal recorded from the fourth $\mathrm{ADC}$ channel is used to analyze the noise in the frequency range $(1-75) \mathrm{Hz}$ and $(2-10000) \mathrm{Hz}$ (Fig. 2).

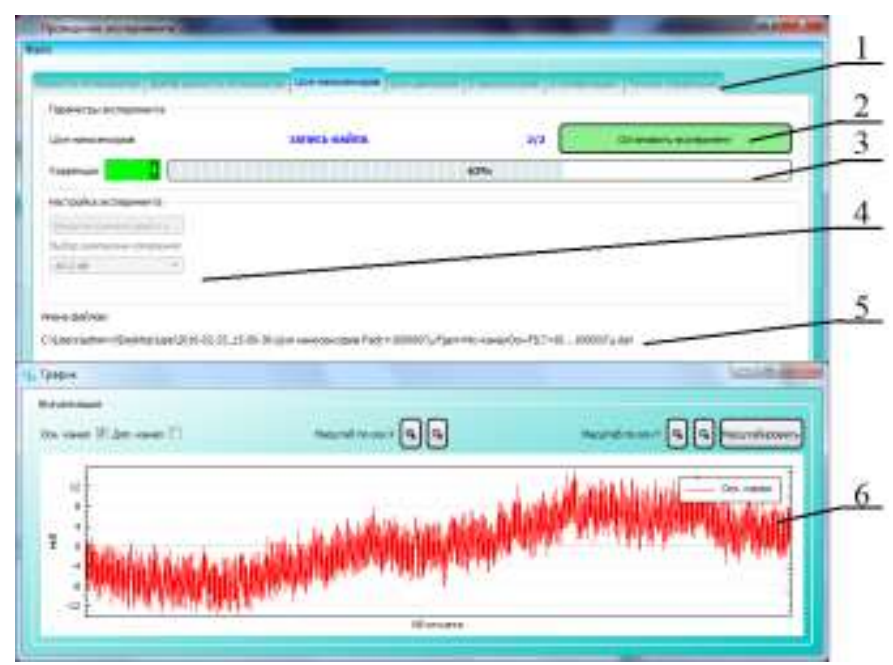

Fig. 2. Interface «Measurement of the electrode noise voltage». 1 - list of experiments, 2 - experiment start/stop button, 3 - experiment progress, 4 experiment settings, 5 - path to output file, 6 - received data 


\section{Measurement of electrodes impedance}

Measurement of electrodes impedance is carried out by an ammeter-voltmeter method therefore current must be supplied to electrode cells. Measuring current passes through the EC. The voltage drop on the EC is read and its amplitude is computed programmatically by the DFT. The voltage divided by the amplitude of the measuring current. Current generator may be external and internal. Internal current generator is controlled by the DAC1. The measurement channel includes amplifiers $\mathrm{A} 1$ and $\mathrm{A} 2$ with the overall gain $\mathrm{Kg} \Sigma=\mathrm{Kg} 1 \mathrm{Kg} 2=$ $100 \cdot 1=100$. In this mode, the program scans only the second ADC channel and outputs the measurement result of electrodes impedance. Measurement range $- \pm 0,2 \mathrm{mV} ; \pm 2 \mathrm{mV} ; \pm 10 \mathrm{mV}$; $\pm 20 \mathrm{mV} ; \pm 100 \mathrm{mV}$, measurement current $-0,1 \mu \mathrm{A} ; 1 \mu \mathrm{A} ; 10$ $\mu \mathrm{A}$, frequency of measurement current $-0,01 \mathrm{~Hz} ; 0,05 \mathrm{~Hz}$; $0,15 \mathrm{~Hz} ; 1 \mathrm{~Hz} ; 2 ; 75 \mathrm{~Hz} ; 10 \mathrm{kHz}$. (Fig. 3).

Cross-platform application framework Qt is the software foundation of the HSCRME. Qt uses standard C++ with extensions including signals and slots that simplifies handling of events. The presence of a vast number of data processing and data visualization tools, and the convenience and relative simplicity of user interface development determined the choice of this package. QCustomPlot library is a Qt C++ widget for plotting and data visualization. Creating an interface template based on the list of tabs is implemented by tabWidget. A fast Fourier transform algorithm is used in this application with help of FFTW library. FTD2XX drivers allow direct access to the USB device through a DLL. Software implementation of band pass filters does not cause much difficulty because the measurement of the electrodes is made in infra- and low range frequency.

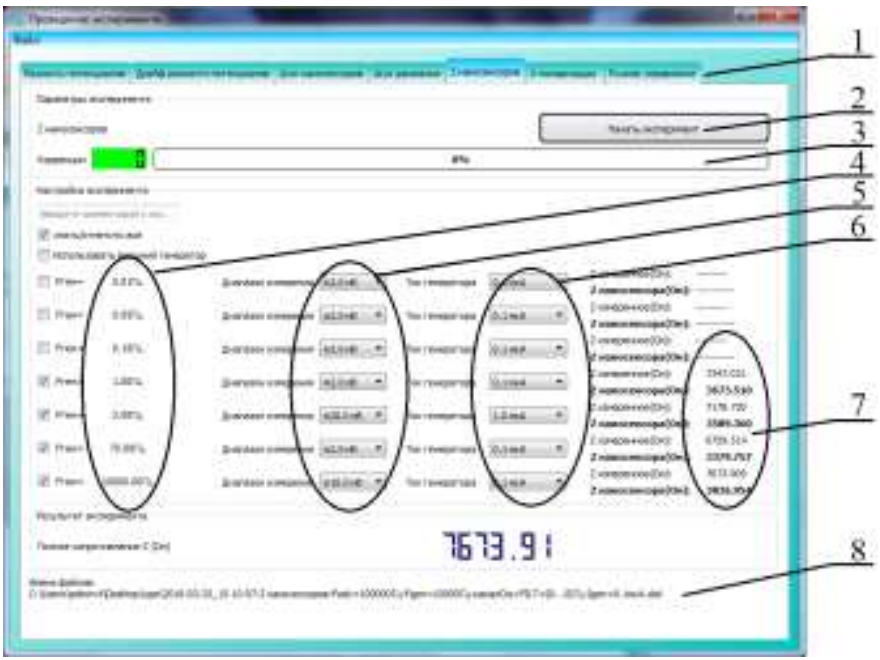

Fig. 3. Interface «Measurement of electrodes impedance ». 1 - list of experiments, 2 - experiment start/stop button, 3 - experiment progress, 4 - list of frequencies, 5 - measurement range, 5 - current, 6 - calculated impedance, 8 - path to output file.

Potential possibilities of the HSCRME are determined by the noise of AMC. The noise amplitude applied to the input of AMC did not exceed $1 \mu \mathrm{V}$ (rms) in the frequency range $0 \ldots 10$ $\mathrm{kHz}$ when instrumentation amplifier AD8422 was used as an input amplifier A1. Verification of the complex was carried out by the inductive voltage divider, which is part of State Standard GAT193-2011 [9, 10]. This State Standard is stored in VNIIFTRI (Moscow).

General view of the HSCRME is shown on fig. 4.

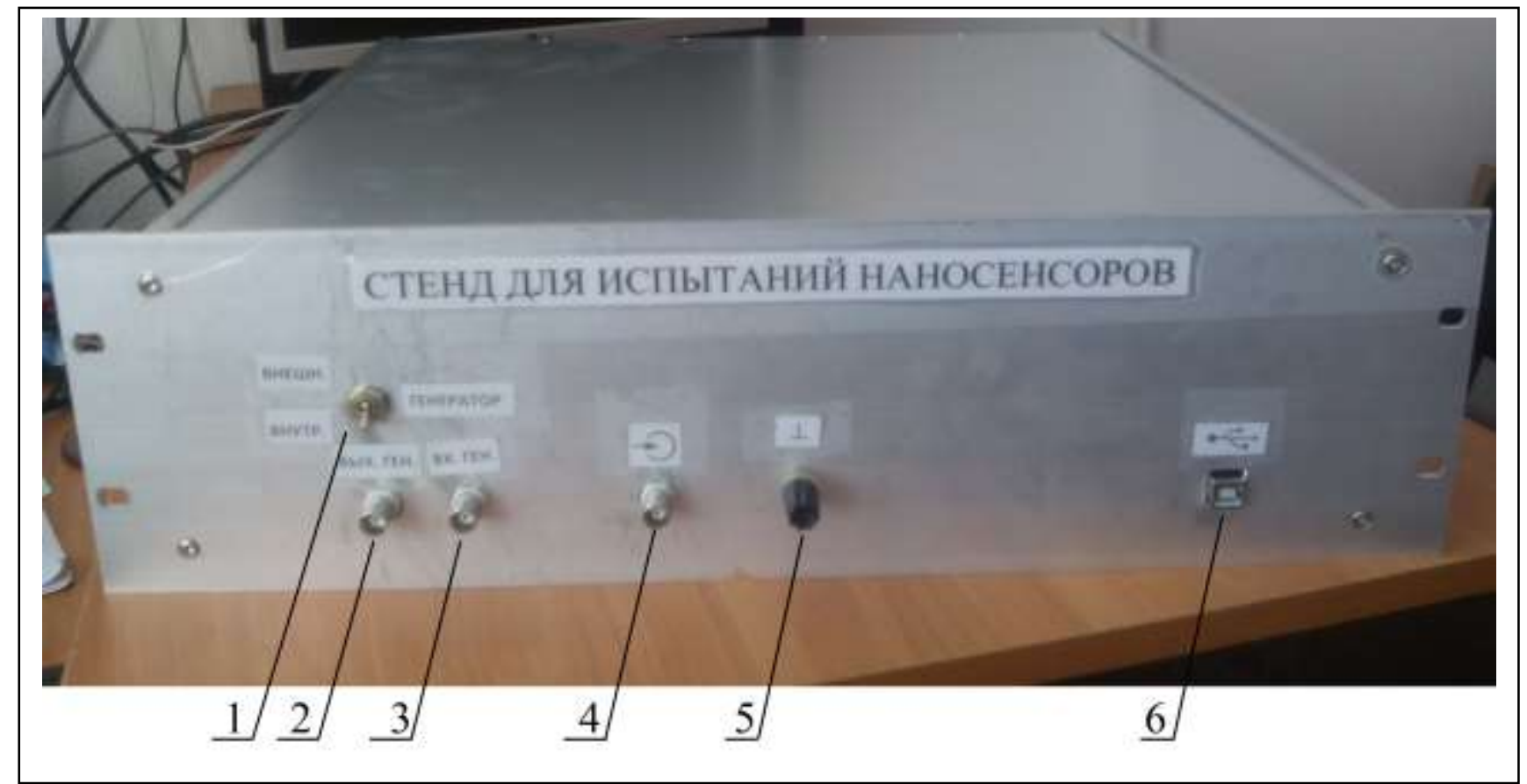

Fig. 4. Appearance of the HSCRME. 1 - switch "external and internal oscillator", 2 - external oscillator output, 3 - external oscillator input, 4 - electrode cells input, 5 - ground, 6 - USB 


\section{BASIC TECHNICAL CHARACTERISTICS OF THE HSCRME}

- Measurement range of electrode potential, $\mathrm{mV}$ $\pm 100$

a) Limit of relative tolerance, $\%- \pm 5$;

b) Input current, $\mathrm{nA}$ - no more than 1.

- Range of the drift of the electrode potential difference measurement, $\mu \mathrm{V}- \pm 150$;

a) Limit of relative tolerance, $\%- \pm 5$;

- Measurement range of noise voltage and electromechanical noise, $\mu \mathrm{V}- \pm 150$;

a) Limit of relative tolerance, $\%- \pm 5$;

- Measurement range of electrodes impedance, $\mathrm{kOhm}-$ $0,05 \ldots 10$;

a) Limit of relative tolerance, $\%- \pm 5$;

b) Measurement current, $\mu \mathrm{A}-0,1 ; 1 ; 10$;

c) Frequency of measurement current, $\mathrm{Hz}$ 0,$01 ; 0,05 ; 0,15 ; 1 ; 2 ; 75 ; 10000$.

- Measurement range of polarization voltage, $\mathrm{mV}$ \pm 25 ;

a) Limit of relative tolerance, $\%- \pm 5$;

b) Measurement current, $\mu \mathrm{A}-0,1$.

\section{CONCLUSION}

The developed complex in conjunction with the technical and program documentation provides:

1) conditions for implementing this complex in the research and industrial organizations involved in the development and creation of a new generation of medical devices with improved diagnostic capabilities;

2) design error elimination of medical devices in the early design stages by rejecting faulty electrodes;

3) creation of metrological basis for the development and production of new medical electrodes, including nanoelectrodes;

4) creation of experimental basis for further training in the field of medical equipment, information and measuring equipment, computer-aided science engineering, automation of measurements, control and diagnostics;

5) an opportunity for Russian manufacturers to obtain high-quality medical electrodes that will be competitive with products of the leading foreign companies;
6) carrying out expert examination and tests when the type approval procedure of imported foreign electrodes take place.

\section{ACKNOWLEDGMENT}

The research was financially supported by the Federal Targeted Programme "Research and Development in Priority Fields of S\&T Complex of Russia in 2014-2020", the Agreement No. 14.578.21.0032 dated 05.06.2014 "Development of the experimental sample of a hardwaresoftware complex for noninvasive recording of heart micropotentials in a wide frequency band without filtering and averaging in real time to early detect the symptoms of a sudden cardiac death"; the unique identifier of the contract: RFMEF157814X0032.

\section{REFERENCES}

[1] Wilhelm J. Kaiser, Horst Weber, Wolfgang Winter. Method and Apparatus for Reducing Noise and Detecting Electrode Faults in Medical Equipment. U.S. Patent 2002/0183797 A1.

[2] Paterson, William G., Blaha, Derek M. Method and System for Continuity Testing of Medical Electrodes. EU Patent 2005/1566645 A2.

[3] Stéphane Bibian, Tatjana Zikov. Method and System for Electrode Impedance Measurement. U.S. Patent 2011/0295096 A1.

[4] D.K. Avdeeva, O.N. Vylegzhanin, I.S. Grekhov, et al. Experimental Results of Electric Activity of «electronic-ionic conduction» Junction. - European journal of natural history, 2009, No 2, P. 98.

[5] D.K. Avdeeva, O.N. Vylegzhanin, I.S. Grekhov, et al. Automatic Device for Testing Medical Electrodes. - Biomedical Engineering, Vol. 43 (2009), No. 2, P. 51-55.

[6] Hilberer E. Evaluation circuit for thick film pressure sensor for ohmic measuring bridge. DE Patent 1997/19531386 A1

[7] Kubota Y, Katoh K., Yoneda H. Signal amplifier, signal amplifier circuit, signal line drive circuit and image display device. U.S. Patent 2000/6054976 A

[8] Shimokawa R., Ueda S., Kamata Y., Yamashita K., Kato K., Sato H. Amplifier and display using the former. U.S. Patent 1988/4755768 A

[9] V.L. Kim: Inductive Voltage Divider. Basics, concept, methods, application. - Saarbrücken: Palmarium Academic Publishing, 2012. $258 \mathrm{p}$.

[10] V.L. Kim, D.S. Cheburenko, S.V. Merkulov: Programmable Inductive Voltage Divider - 7th International Forum on Strategic Technology (IFOST 2012): Proceedings: in 2 vol., Tomsk, September 18-21, 2012. - Tomsk: TPU Press, Vol. 1 (2012), p. 624-627. 